\title{
Is it sustainable? A comparison of student and tutor online time across three distance-learning
}

\section{courses}

\author{
Tina Wilson* and Denise Whitelock** \\ *Centre for Educational Software, Open University \\ **Institute of Educational Technology, Open University.
}

Distance-learning course providers world-wide are looking to new technology to enhance their course presentations. One of the areas they are beginning to assess is that of integrating Computer Mediated Communication (CMC) into their courses. An important factor to be borne in mind with the adoption of $C M C$ is the amount of time required to be an active participant. It is important therefore to investigate how much time tutors and students spend online, and if this is sustainable in the longer term. This paper discusses tutor and student involvement in the Open University M205 - STILE project, and compares these findings with the time spent online by students and tutors on two other Open University courses. Our aim was to see if our findings were atypical. The comparisons are discussed, and recommendations are made about how future online courses can benefit from the findings of this study.

\section{Introduction}

Computer Mediated Communication (CMC) is a useful pedagogical tool. Mason (1989a) states that it has proved to be 'a life-line to help, information, contact and exchange' for students. Thomas (1989) discusses the benefits of CMC from the tutor and course team perspectives. However, Rumble (1989) discusses the difficulties of costing the introduction of $\mathrm{CMC}$ into a course. If more and more distance-learning institutions are going to adopt this medium, it is important to evaluate for all concerned the time required for this type of participation.

Projects which have monitored the time requirement for online working by students and tutors are essential to inform this debate. STILE (Students' and Teachers' Integrated Learning Environment) is one such project which was undertaken at four universities in the United Kingdom. The Open University's implementation of the project, called M205 STILE, used a CMC environment, in addition to the World Wide Web (see Wilson and Whitelock, 1997a and b; for more information on the M205 STILE environment, see Wilson and Whitelock, 1996 and 1997c), with 110 students and nine tutors, nation-wide 
and in Europe. The participants were online for a period of nine months in 1995. This paper reports on patterns of usage in the M205 - STILE CMC environment with respect to the nature of student-tutor interactions and time spent online. In a further analysis of these findings, a comparison is made with two other Open University courses, in order to ascertain whether our findings are atypical. This paper thus presents a range of empirical findings which address:

- how much time students spent online;

- how much time tutors spent online;

- this compared with two other courses which also used CMC.

\section{Results}

The students at the Open University were taking the Fundamentals of Computing (M205) course which catered for both technical and non-technical students. At the start of 1995, 110 students successfully logged into the M205 - STILE environment.

\section{Time spent online by the students}

At the end of the course, we were interested to know how long individuals had actually spent online, especially as they did not have an offline reader available to them in 1995. As a group, the M205-STILE students spent a total of 934 hours online. As an average, each student spent 8.5 hours online, but the amount of time spent online varied quite considerably between students. Some 15 per cent spent less than two hours online, approximately half spent less than six hours online, and a small minority ( 5 per cent) spent more than 24 hours online (see Figure 1). •

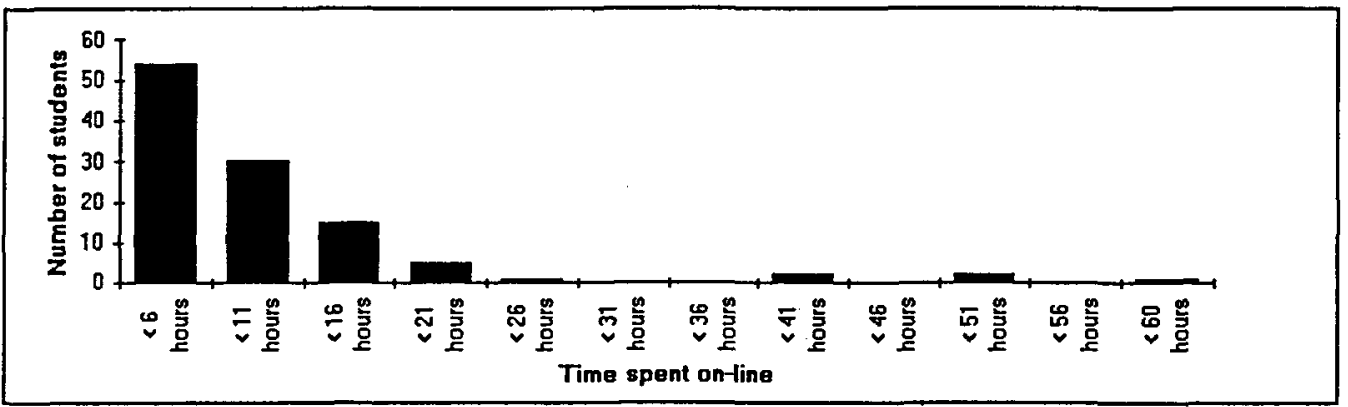

Figure I: Time spent online by students

As approximately half of the students spent less than six hours online over a nine-month period, it is not surprising that they did not have sufficient time to read messages, and to post messages to ask for help in the different conferences (for information about when the students were available on a month-by-month basis, see Wilson and Whitelock, 1997d). The figures are interesting in themselves, but may well show a disappointing result with respect to usage of this expensive learning aid. Therefore, to understand if our group of students displayed an atypical amount of time online, we compared our data with other courses which had used online conferencing (see Table 1). Both DT200, 'Introduction to 
Information Technology' and B885, 'The Challenge of the External Environment', used conferencing. Although they used a different conferencing system, they, like the M205 STILE students, did not have an offline reader. Their participation in the online presentation was largely optional (as was the case with M205-STILE), but both DT200 in 1989 and B885 in 1992 awarded marks for online use of the conferencing system. On DT200 Mason (1989) mentions that ' 10 per cent of the marks on one assignment were given for conference and mail entries'. On B885 Pearson and Mason (1992), mention that 'marks were awarded for both team (group) and individual use of computer conferencing in TMA 02'. M205 - STILE's use of FirstClass was optional and marks could not be awarded for online use because the 110 students involved could have been seen as having an advantage when compared with the other 3,000 or more students taking the usual presentation of the course.

If we compare the time spent online by M205-STILE students and DT200 students with B885 we find a marked difference in the number of students who did not spend any time online. All of the M205 students went online, approximately 90 per cent of the DT200 students participated but only 67 per cent of the B885 students actually went online. One reason for this could be that $\mathrm{B} 885$ was a postgraduate course and these students did not feel as much need for this facility. Looking at the 1-2 hour period it is interesting that twice as many M205-STILE students used the online facilities when compared with the DT200 students. However the 2-3 hour range has fairly similar percentages of students using the online environment across all three courses.

\begin{tabular}{lcccc}
\hline Hours & $\begin{array}{c}\text { DT200 '88* } \\
\text { \% students }\end{array}$ & $\begin{array}{c}\text { DT200 '89* } \\
\text { \% students }\end{array}$ & $\begin{array}{c}\text { B885 '92*** } \\
\text { \% students }\end{array}$ & $\begin{array}{c}\text { M205 - STILE '95 } \\
\text { \% students }\end{array}$ \\
\hline None & 8.4 & 11 & 32.8 & 0 \\
Up to I hour & 5.3 & 5.6 & 13 & 4.5 \\
I-2 hours & 7 & 7.1 & 11.1 & 14.5 \\
2-3 hours & 9.1 & 8 & 8.3 & 10 \\
3-5 hours & 15.6 & 17.4 & 9.8 & 15.4 \\
5-10 hours & 27.9 & 33.1 & 15.4 & 29.1 \\
10-20 hours & 19 & 13.8 & 6.7 & 21 \\
20-30 hours & 4.3 & 2.8 & 2.2 & 0.9 \\
30-40 hours & 1.8 & 0.6 & 0.7 & 0.9 \\
Over 40 hours & 1.4 & 0.6 & 0 & 3.6 \\
\hline
\end{tabular}

* Mason (1989a and b) converted to percentages.

**Pearson and Mason (1992).

Table 1: A comparison of time spent online by students on other courses with M205 - STILE

If we look at the time range 5-10 hours across all three courses, we see that this range has the highest percentage of students using the online facilities in each case. This suggests that the 5-10 hour period could be the amount of time which students on all three courses would agree is necessary for optional participation in a CMC. However, if we group the 5-10 and 10-20 time ranges together, we achieve a markedly different result. These time 
ranges taken together account for 47 per cent of the students studying DT200, and 50 per cent of the M205 - STILE students, but only 22 per cent of the B885 students. This suggests that undergraduate students may elect to spend between 5 and 20 hours online, while postgraduate students might elect for only 5 to 10 hours. Our findings complement those of Mason (1989a and b) for DT200 in most time ranges. However, the higher percentage of students who used M205 - STILE for over 40 hours could be an indication that these students found the environment very motivating.

Time spent online by the tutors

At a meeting before the project started, the tutors taking part in M205 - STILE were advised to spend on average one hour per week, over 30 weeks, on the online part of the presentation. This was to include necessary work offline, for example checking program code, looking up a query or responding to points raised. It was stressed that it was likely that they would probably need to spend more time online at the beginning of the course while the students were becoming acquainted with the new medium. The tutors collectively spent a total of 192 hours online. This averages out to 21 hours per tutor, so the ratio of tutor-time to student-time was approximately three to one. These metrics require further investigation on other courses, but if tutors continue to use the environment on average three times as much as the students, we must ask if this is sustainable in terms of staff costs. Strategies need to be developed to improve the ratio of tutor-to-student online time. As with the students, however, the amount of time spent online by the tutors varied quite considerably, here with a minimum of 12 hours and a maximum of 43 (see Figure 2).

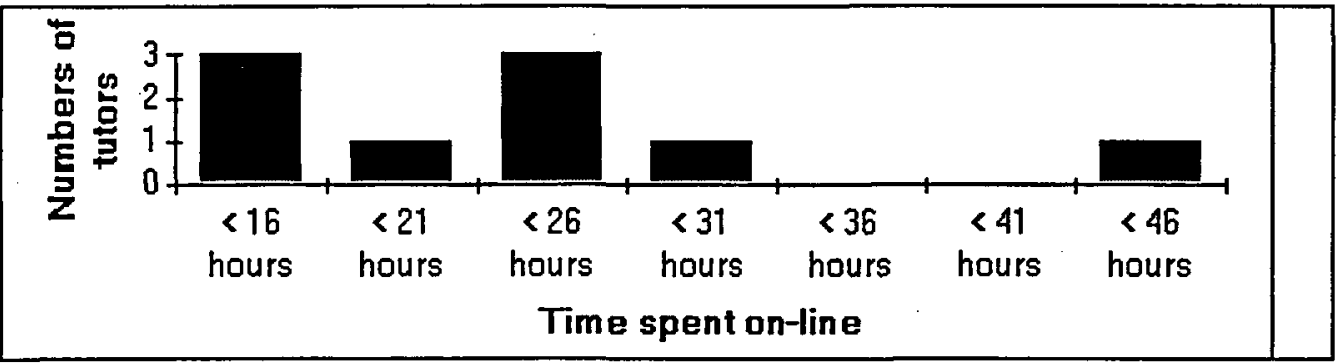

Figure 2: Time spent online by tutors

Again, these figures are interesting in themselves, but to understand if our tutors spent an atypical amount of time online, we once more compared our data with DT200 and B885 which had used online conferencing (see Table 2). Unfortunately because of the different time ranges used by Mason (1989) and Pearson and Mason (1992), we could only make a comparison between quite broad time bands. On the DT200 course the tutors were advised to spend 8 hours of tutorial time in the online part of the presentation. It was not clear if the B885 tutors were guided about how long to spend online. It's interesting however that the majority of tutors on DT200 and B885 were online for more than 3 hours but less than 20 hours while the M205-STILE tutors were equally split between the time bands of 3-20 hours and 20-30 hours. This contrasts with a high percentage of B885 tutors, some 28.14 per cent who spent less than 3 hours online. 
ALT-J Volume 6 Number 1

\begin{tabular}{lcccc}
\hline Hours & $\begin{array}{c}\text { DT200 '88* } \\
\text { \% tutors }\end{array}$ & $\begin{array}{c}\text { DT200 '89* } \\
\text { \% tutors }\end{array}$ & $\begin{array}{c}\text { B885 '92 ** } \\
\text { \% tutors }\end{array}$ & $\begin{array}{c}\text { M205 - STILE '95 } \\
\text { \% tutors }\end{array}$ \\
\hline Up to 3 hours & 1.5 & 3.2 & 28.14 & 0 \\
3-20 hours & 66.2 & 74.3 & 56.25 & 44.44 \\
20-30 hours & 9.2 & 11.3 & 3.13 & 44.44 \\
Over 30 hours & 23.1 & 11.3 & 12.5 & 11.11 \\
\hline
\end{tabular}

* Mason (1989b), converted to percentages.

**Pearson and Mason (1992).

Table 2: A comparison of time spent online by tutors on other courses with M205 - STILE

In $1988,23.1$ per cent of the DT200 tutors spent more than 30 hours online. In 1989 this dropped by half, suggesting that in the first year of presentation tutors perceived a need to be available online more often. The fact that 44.4 per cent of the M205 - STILE tutors were online for over 20 hours but under 30 hours in 1995 might be explained by the initial expectation that they would be online for one hour for each of the 30 weeks of the course. This might also explain why only 11.1 per cent of the M205-STILE tutors were online for over 30 hours. The DT200 tutors, by contrast, had been expected to spend eight hours in total online over the 30-week period. Although we cannot look at the eight-hour break point, we can see in the bands $20-30$ and over 30 hours that some tutors found it hard to keep to this restriction.

\section{Conclusions}

Levels of student participation on M205 - STILE were fairly typical of an undergraduate course. However, a higher percentage of students used the system for more than 40 hours. It will therefore be necessary to devise ways of working online and offline to save on telephone costs and time for these students. When we compare the online time across the three courses, the figures suggest that students feel it necessary to spend between 5 and 10 hours online for optional participation. But a high percentage of undergraduate students taking DT200 and M205 - STILE felt it was necessary to spend between 10 and 20 hours online. Although the award of marks for online participation was introduced to DT200 for the second presentation of the course in 1989, the amount of time spent online was not significantly different from that of 1988 . When we look at the time spent online by the DT200 students in 1989 and the B885 students in 1992, when both courses were awarding marks for participation, and compare these with M205 - STILE (where no marks were awarded), it is interesting that the M205 - STILE students as a group actually spent more time online. This suggests that the M205 - STILE students found the online environment valuable even though they were not awarded marks for participation.

CMC raises expectations where students anticipate an immediate response from their tutors because they are used to the immediacy of the telephone and other methods of engagement. In M205 - STILE, tutor participation was high with 44.4 per cent spending between 20 and 30 hours online, thus on average at a 3:1 ratio when compared with the students' average online time. This ratio cannot be sustained, and strategies need to be 
developed to improve it: further investigation is required into how much time tutors need to spend in online activities.

Although the tutors were available, some students still perceived that their tutor was not available enough or when they wanted them to be. One student commented that tutors seemed to $\log$ on only once or twice a week and usually at the weekends. This suggests that student perceptions and expectations of an instant response is higher in an electronic environment. Indeed Rumble (1989) alludes to the necessity of 'change and new practices having to be adopted'. Perhaps the tutors should be asked what they think is a reasonable amount of online time in order for them to facilitate learning. However, it must be borne in mind that if they are being paid on an hourly basis for this activity, this could influence their response.

Perhaps the amount of time spent online by tutors could be dramatically reduced if the tutors developed a method for online working and built up a database of answers to frequently asked questions. The introduction of an offline reader may also reduce the amount of time spent online, but not necessarily the amount of time answering queries. The number of tutors on DT200 who spent more than 30 hours online dropped by half in the second year of presentation, suggesting that tutors were more used to using the medium in the second year. Again, further research is required with new courses which adopt CMC to see if the tutors are learning how to use the medium in the first year of presentation, and if the time spent online is dramatically reduced as a result in the second year. In addition, guidelines are required for how much time tutors should spend online. It should be suggested to the tutors that they inform the students about what the latter can reasonably expect; that is, how quickly students can expect a response, and how often they themselves will be online each week. Then the students can gauge when it will be beneficial to post messages online. The comparisons across the three courses suggest that tutors perhaps need to be online for more than eight hours but not as many as $\mathbf{3 0}$ hours. A more suitable length of time would perhaps be 15 hours. This would almost double the suggested DT200 online time, but half that of M205-STILE.

\section{Acknowledgements}

We thank the nine tutors involved in M205-STILE. The research described in this paper was undertaken in the Computing Department, Faculty of Mathematics and Computing, at the Open University as part of the STILE project which was supported by a grant from the Teaching and Learning Technology Programme.

\section{References}

Mason, R. (1989a), 'An evaluation of CoSy at the Open University' in Mason, R. and Kaye, A. (eds), Mindweave, Oxford: Pergamon Press.

Mason, Robin (1989b), Use of CoSy on DT200, 1989, Open University CITE Report No. 99.

Pearson, J. and Mason, R. (1992), An Evaluation of the Use of CoSy on B885 in 1992, Open University CITE Report No. 171. 
Rumble, G. (1989), 'Online costs: interactivity at a price' in Mason, R. and Kaye, A. (eds), Mindweave, Oxford: Pergamon Press.

Thomas, R. (1989), 'Implications of electronic communication for the Open University' in Mason, R. and Kaye, A. (eds), Mindweave, Oxford: Pergamon Press.

Wilson, T,. and Whitelock, D. (1996), 'Piloting a new approach: making use of new technology to present a distance-learning Computer Science course', $A L T-J, 4$ (1).

Wilson, T. and Whitelock, D. (1997a), 'Hijacking hypermedia and other highways to learn computer science on a distance-learning course', $A L T-J, 5$ (2).

Wilson, T. and Whitelock, D. (1997b), 'Opening up horizons: providing online course material in cyberspace' in Travis, D. (ed), Special issue of the Displays Journal on Multimedia, 17 (3-4).

Wilson, T. and Whitelock, D. (1997c). 'Facilitating electronic communication: evaluating Computer Science tutors' and students' interaction using computer-mediated communication at a distance-learning university' in Cornell, $\mathrm{R}$. and Ingram, $\mathrm{K}$. (eds), $A n$ International Survey of Distance Education and Training for Organizations: From Smoke Signals to Satellite III, A Report for the International Council for Educational Media (ICEM), 74-94. Presented at the ICEM Media Week in Berlin, March 1997.

Wilson, T. and Whitelock, D. (1997d), 'To collaborate or not to collaborate? Monitoring the online antics of distance-learning Computer Science students', Proceedings of the Eighth International PEG Conference, Sozopol, Bulgaria. 30 May-1 June 1997. 